\title{
NMR in Life Sciences
}

\author{
K. M. Salikhov
}

Published online: 22 March 2014

(C) Springer-Verlag Wien 2014

Nuclear magnetic resonance spectroscopy is widely used for studying molecular structure of compounds, molecular mobility, kinetics of chemical reactions, etc. It is impossible to overestimate the contribution of methods developed in nuclear magnetic resonance spectroscopy to solving problems of molecular biology and medicine, and to medical diagnostics with the help of magnetic resonance tomography. Different paramagnetic additives (contrast agents) are used to obtain images in magnetic resonance tomography. These additives should meet a series of requirements. The most important requirement in medical applications of magnetic resonance tomography is the safety of contrast agents for a human organism. It is desirable that these paramagnetic additives be concentrated in a site-directed manner in human body. They should also affect the proton longitudinal and transverse relaxation times in a controlled manner.

It is my pleasure to present the authors of this special issue of Applied Magnetic Resonance and to comment in short about the content of the papers devoted to interesting examples of applications of methods of nuclear magnetic resonance spectroscopy.

M.A. Shevtsov, B.P. Nikolaev, Ya.Yu. Marchenko, L.Y. Yakovleva, A.V. Dobrodumov, G. Török, E. Pitkin, and T.V. Lebedev studied water-soluble gadofullerene Gd@Ful. The Gd@Ful nanosuspension was shown to be a contrast enhancer with high anti-tumor therapeutic potency. Yu.V. Bogachev, Ju.S. Chernenco, K.G. Gareev, I.E. Kononova, L.B. Matyushkin, V.A. Moshnikov, and S. S. Nalimova investigated the aggregation processes in colloidal solutions of magnetite-silica nanoparticles. It was shown that the synthesized nanoparticles can be used as MRI contrast agents.

\footnotetext{
K. M. Salikhov ( $\bowtie)$

Zavoisky Physical-Technical Institute, Russian Academy of Sciences, Sibirsky Trakt, 10/7, Kazan 420029, Russian Federation e-mail: salikhov@kfti.knc.ru
} 
The study performed by M.V. Popova, Y.S. Chernyshev, and D. Michel showed that ${ }^{23} \mathrm{Na}$ relaxation measurements can be a valuable tool for determining the critical micelle concentration, second critical micelle concentration and critical aggregation concentration on the surface. M.V Popova and D. Michel studied sodium lauroyl sarcosinate (SLAS) and observed the trans and cis-isomers of SLAS in solution.

At present, a great deal of attention has been paid to the development of targeted drug delivery systems. Porous materials can be used as drug containers. In general, porous materials are of interest from different points of view. V.A. Ryzhov, I.V. Pleshakov, A.A. Nechitailov, N.V. Glebova, E.N. Pyatyshev, A.V. Malkova, I.A. Kiselev, and V.V. Matveev investigated a magnetically ordered material, a composite structure obtained by embedding of cobalt-containing substance into pores of silicon matrix. To study the self-diffusion of silicone MQ copolymers in solutions with chloroform over a wide range of macromolecular concentrations S.G. Vasil'ev, V.I. Volkov, E.A. Tatarinova, and A.M. Muzafarov used pulsed-fieldgradient nuclear magnetic resonance.

This special issue of Applied Magnetic Resonance was edited by Professors Vladimir I. Chizhik (St. Petersburg, Russia) and Murat S. Tagirov (Kazan, Russia). They did a great job and I am most appreciative of their time and efforts. Special thanks go to Associate Professor Marina G. Shelyapina (St. Petersburg, Russia).

Kev Salikhov

Editor-in-chief 